\title{
Collective Treatment of the Giant Resonances in Spherical Nuclei*
}

\author{
M. G. Huber $\dagger$ and M. Danos \\ National Bureau of Standards, Washington, D. C. \\ AND \\ H. J. WeBER AND W. GREINER \\ Institut fuer Theoretische Physik, University of Frankfurt, Frankfurt, Germany
}

(Received 27 July 1966)

\begin{abstract}
In a collective treatment the energies of the giant resonances are given by the boundary conditions at the nuclear surface, which is subject to vibration in spherical nuclei. The general form of the coupling between these two collective motions is given by angular-momentum and parity conservation. The coupling constants are completely determined within the hydrodynamical model. In the present treatment the influence of the surface vibrations on the total photon-absorption cross section is calculated. It turns out that in most of the spherical nuclei this interaction leads to a pronounced structure in the cross section. The agreement with the experiments in medium-heavy nuclei is striking; many of the experimental characteristics are reproduced by the present calculations. In some nuclei, however, there seem to be indications of singleparticle excitations which are not yet contained in this work.
\end{abstract}

\section{INTRODUCTION}

$I^{\mathrm{T}}$ $\mathrm{T}$ is known from the location of the low-energy states and from the magnitudes of the $E 2$ transition probabilities between these states that in the vibrational nuclei the shape oscillations have rather large amplitudes. ${ }^{1}$ In fact, very frequently the mean-square deformation associated with the surface vibrations is comparable in magnitude with that of the permanently deformed nuclei. ${ }^{2}$ The interaction of the giant resonance oscillation with the surface vibrations, therefore, must be expected to be very important. In contrast to the heavy deformed nuclei, where a static treatment of the nuclear surface already leads to a qualitatively correct description of the splitting of the giant resonance, ${ }^{3,4}$ here the interaction is totally a dynamic effect, viz., that of coupled oscillators. ${ }^{5,6}$ Also, because of the large amplitudes of the surface vibrations, it should be expected that a treatment considering these vibrations to be harmonic will have only limited accuracy. However, a power-series expansion in terms of the vibrational amplitudes should converge quite rapidly.

The need to go beyond the harmonic approximation is evident from the low-energy spectra. In even-even nuclei the two-photon states should form a degenerate triplet $0^{+}, 2^{+}, 4^{+}$at an energy twice that of the first excited $2^{+}$state. Instead, in many nuclei there are more

* Work supported in part by the German Bundesministerium fuer Wissenschaftliche Forschung and the Deutsche Forschungsgemeinschaft.

$\dagger$ On leave of absence from the University of Frankfurt, Frankfurt, Germany.

${ }^{1}$ A. Bohr and B. R. Mottelson, Kgl. Danske Videnskab. Selskab, Mat. Fys. Medd. 27, 16 (1953).

${ }_{2}^{2}$ P. H. Stelson and L. Grodzins, Nucl. Data 1, 21 (1965).

${ }^{3}$ M. Danos, Nucl. Phys. 5, 23 (1958); K. Okamoto, Phys. Rev. 110, $143(1958)$.

${ }^{4}$ M. Danos and W. Greiner, Phys. Rev. 138, B1055 (1965)

${ }^{5} \mathrm{~J}$. LeTourneux, Kgl. Danske Videnskab. Selskab, Mat. Fys. Medd. 34, 11 (1965); J. LeTourneux, Phys. Letters 13, 325 (1964).

${ }^{6}$ M. G. Huber, H. J. Weber, M. Danos, and W. Greiner, Phys. Rev. Letters 15, 529 (1965); M. G. Huber, H. J. Weber, and W. Greiner, Z. Physik 192, 223 (1966). than three states at approximately that energy, and frequently the splitting in the multiplet is of the same order as the harmonic oscillator energy. The experimental spectra are still more confused in odd nuclei. Not much is known about the three-phonon states. It seems worthwhile to undertake a systematic development of the theory and to compare the results with the experimental data at each step in order to obtain information on the actual importance of the different interaction terms.

The present paper has three aims. First, we present a quantum-mechanical treatment of the coupled system of surface vibrations and giant dipole oscillations which encompasses all terms up to second order in the vibrational amplitudes. The form of the Hamiltonian is uniquely determined by angular-momentum and parity considerations. All coupling constants are computed in the adiabatic approximation from the collective model. ${ }^{7}$ The earlier paper by LeTourneux ${ }^{5}$ considers only the lowest order in the interaction. We also go beyond LeTourneux's treatment by including the influence of the surface vibrations on the dipole matrix elements in the absorption cross section. We compare the predictions of the theory with the available experimental data. This part is contained in Secs. II through VI.

Second, we give a series of photon-absorption cross sections computed for different nuclear parameters. They have been chosen such that the complete range of the experimentally observed parameters is covered with a sufficient density. This way it is possible to obtain a "predicted" cross section for any particular nucleus as an interpolation between the given curves. We have done this to facilitate the planning of photonuclear experiments (Sec. VII).

Third, we present a discussion on the limits of validity

${ }^{7}$ T. Urbas, Diplomarbeit, University of Frankfurt, 1966 (unpublished). 
of the theory and on the accuracy of the solutions obtained. This is done in Sec. VIII.

\section{THE HAMILTONIAN}

The Hamiltonian describing the surface quadrupole vibrations, the giant dipole resonances, and the interaction between these two collective motions can be written

$$
H=H_{\text {quad }}+H_{\text {dip }}+H_{\text {dip quad }} .
$$

We discuss the different terms separately. We begin with $H_{\text {quad }}$. The nuclear surface usually is expanded in spherical harmonics. Taking into account only quadrupole deformations the surface is described by

$$
R=R_{0}\left(1+\sum_{\mu} \alpha_{2 \mu} Y_{2 \mu}\right) .
$$

Here the $\alpha_{2 \mu}$ are considered to be time-dependent, $\alpha_{2 \mu}(t)$, and their motion is assumed to be harmonic. Introducing the conjugate momenta

$$
\pi_{2 \mu}=\partial H / \partial \dot{\alpha}_{2 \mu}
$$

and using the notation of angular-momentum coupling of Fano and Racah ${ }^{8}$ the Hamiltonian of these quadrupole vibrations has the following form:

$$
\begin{aligned}
H_{\text {quad }}=\left(\frac{1}{2} \sqrt{ } 5\right)\left(B_{2}{ }^{-1}\left[\pi^{[2]} \times \pi^{[2]}\right]^{[0]}\right. & \left.+C_{2}\left[\alpha^{[2]} \times \alpha^{[2]}\right]^{[0]}\right) .
\end{aligned}
$$

The harmonic approximation describes the lowenergy properties in the spherical even-even nuclei to some extent. However, for instance, the two-phonon states are observed to be nondegenerate in almost all vibrational nuclei. To describe this, anharmonic terms would have to be added to Eq. (3); we neglect them at this time.

The giant dipole resonances can be understood in terms of protons and neutrons vibrating against each other because of a potential of the form

$$
V=K \int \rho_{0}^{-1}\left(\rho_{p}-\rho_{n}\right)^{2} d V
$$

Assuming constant total density we write for the proton and neutron densities

$$
\begin{aligned}
\rho_{p} & =\frac{Z}{A} \rho_{0}\left[1+\eta(r) e^{-i \omega t}\right], \\
\rho_{n} & =\frac{N}{A} \rho_{0}\left[1-\frac{Z}{N} \eta(r) e^{-i \omega t}\right] .
\end{aligned}
$$

Here $\rho_{0}$ is the mass density distribution of the nucleus. The spatial part of the function $\eta(r)$ is determined

${ }^{8}$ U. Fano and G. Racah, Irreducible Tensorial Sets (Academic Press Inc., New York, 1959). by the Helmholtz equation ${ }^{9}$

with

$$
\Delta \eta+k^{2} \eta=0
$$

$$
\begin{aligned}
& k^{2}=\omega^{2} / u^{2}, \\
& u^{2}=(8 K / M)\left(N Z / A^{2}\right),
\end{aligned}
$$

and $K$ is the symmetry energy parameter of the BetheWeizsäcker formula. The deviation density is written as

$$
\eta(r, t)=\sum_{\lambda_{\nu}} C_{\lambda_{\nu}} \alpha_{\lambda_{\nu}}(t) j_{\lambda}\left(k_{\lambda} r\right) Y_{\lambda_{\nu}}(\hat{r}) .
$$

The $C_{\lambda_{\nu}}$ and $\alpha_{\lambda \nu}$ are normalization constants and amplitudes of the different modes of motion, respectively. We will restrict ourselves to the dipole case, i.e., $\lambda=1$.

The energies of the different modes are determined by the condition that the radial flux vanish on the nuclear surface. Thus the energy is determined by the relation

$$
k_{n} R=z_{n},
$$

where $z_{n}$ is the $n$th zero of the derivative $j_{1}{ }^{\prime}(z)$. These zeros are well known ${ }^{9}$ to be 2.08, 5.95, 9.20 for $n=1,2,3$, respectively.

To go from classical to quantum hydrodynamics, the amplitudes $\alpha_{1 \nu}$ are taken to be operators. Introducing conjugate momenta $\pi_{1 \nu}$, the Hamiltonian of this collective motion is given by

$H_{\mathrm{dip}}=-\frac{1}{2} \sqrt{3}\left(B_{1}^{-1}\left[\pi^{[1]} \times \pi^{[1]}\right]^{[0]}+C_{1}\left[\alpha^{[1]} \times \alpha^{[1]}\right]^{[0]}\right)$.

The coupling between the surface and the giant dipole oscillations arises via the boundary conditions.

According to Eq. (9) the energy of the dipole resonance is determined by the nuclear surface, Eq. (2), which in turn depends on the collective variables of the quadrupole oscillations, $\alpha_{2 \mu}$. Therefore the total Hamiltonian contains interaction terms. From angular momentum and parity considerations the interaction Hamiltonian must be of the following form:

$$
\begin{aligned}
& H_{\text {dip quad }}=K_{1}\left[\alpha^{[2]} \times \alpha^{[1]} \times \alpha^{[1]}\right]^{[0]} \\
& \quad+K_{20}\left[\alpha^{[2]} \times \alpha^{[2]}\right]^{[0]}\left[\alpha^{[1]} \times \alpha^{[1]}\right]^{[0]} \\
& \quad+K_{22}\left[\left[\alpha^{[2]} \times \alpha^{[2]}\right]^{[2]} \times\left[\alpha^{[1]} \times \alpha^{[1]}\right]^{[2]}\right]^{[0]}+\cdots .
\end{aligned}
$$

The coupling constants can be evaluated in the adiabatic approximation which here can be expected to give very good results. In the even-even spherical nuclei the ratio of the energies of the giant dipole resonance $E_{1}=\hbar \omega_{1}$, and the quadrupole phonons $E_{2}=\hbar \omega_{2}$, lies between 10 and 30 . That means that the nuclear shape does not change appreciably during one period of the dipole oscillation. Therefore the frequencies of the dipole oscillation can be computed as if the nucleus were statically deformed. This deformation leads to a splitting of the three possible dipole modes as has been shown by Danos and Okamoto. ${ }^{3,4}$

${ }^{9}$ M. Danos, University of Maryland Technical Report No. 221, 1961 (unpublished). 
The energies of the three modes are then given by

$$
K_{\nu}=\left(2.08 / R_{\nu}\right)\left[1+0.88\left(\Delta R_{\nu} / R_{0}\right)\right] .
$$

$R_{\nu}, K_{\nu}$ are the three principal axes and the corresponding wave vectors, respectively.

For practical reasons the dependence of the $K_{\nu}$ 's on the deformation parameters is calculated in the instantaneous intrinsic coordinate system. Such a system can be defined for times which are short compared with the period of the surface vibrations. In order to obtain the coupling constants $K_{1}, K_{20}$, and $K_{22}$ one has to make a transformation back into the laboratory system. This has been done by Urbas. ${ }^{7}$ For the coupling constants he obtains the following values:

$$
\begin{aligned}
K_{1} & =-1.588 C_{1}, \\
K_{20} & =-0.708 C_{1}, \\
K_{22} & =-0.936 C_{1} .
\end{aligned}
$$

In principle the same procedure can also be used for the evaluation of the coupling constants of the higherorder interaction terms. Here we restrict ourselves to the second-order terms in the $\alpha_{2 \mu}$. However, as will be seen later, the higher-order interaction terms as well as the anharmonicity of the surface vibrations very likely give nonnegligible contributions and should also be included. This point is under investigation at this time.

\section{SOLUTION OF THE SCHRÖDINGER EQUATION}

Writing the total Hamiltonian as

$$
H=H^{0}+H_{\text {dip quad }},
$$

$H^{0}$ describing the unperturbed surface and charge vibrations,

$$
H^{0}=H_{\text {dip }}+H_{\text {quad }},
$$

and introducing creation and annihilation operators

$$
\begin{aligned}
& \alpha_{1 \nu}=\left(\hbar \omega_{1} / 2 C_{1}\right)^{1 / 2}\left[d_{\nu}^{\dagger}+(-1)^{\nu} d_{-\nu}\right], \\
& \pi_{1 \nu}=i\left(\hbar C_{1} / 2 \omega_{1}\right)^{1 / 2}\left[(-1)^{\nu} d_{-\nu}{ }^{\dagger}-d_{\nu}\right],
\end{aligned}
$$

$$
\begin{aligned}
\alpha_{2 \mu} & =\left(\hbar \omega_{2} / 2 C_{2}\right)^{1 / 2}\left[\beta_{\mu}^{\dagger}+(-1)^{\mu} \beta_{-\mu}\right], \\
\pi_{2 \mu} & =i\left(\hbar C_{2} / 2 \omega_{2}\right)^{1 / 2}\left[(-1)^{\mu} \beta_{-\mu}{ }^{\dagger}-\beta_{\mu}\right], \\
\omega_{1} & =\left(C_{1} / B_{1}\right)^{1 / 2}, \\
\omega_{2} & =\left(C_{2} / B_{2}\right)^{1 / 2},
\end{aligned}
$$

one immediately obtains

$$
\begin{aligned}
& H^{0}=-\sqrt{3} \hbar \omega_{1}\left(\left[d^{\dagger[1]} \times d^{[1]}\right]^{[0]}-\frac{1}{2} \sqrt{3}\right) \\
& +(\sqrt{ } 5) \hbar \omega_{2}\left(\left[\beta^{\dagger[2]} \times \beta^{[2]}\right]^{[0]}+\frac{1}{2} \sqrt{ } 5\right) .
\end{aligned}
$$

The energies of $H^{0}$ are given by

$$
E\left(N_{1}, N_{2}\right)=\left(N_{1}+\frac{3}{2}\right) \hbar \omega_{1}+\left(N_{2}+\frac{5}{2}\right) \hbar \omega_{2} .
$$

A very important quantity is the mean-square amplitude of the surface vibrations, $\beta_{0}{ }^{2}$, which is in terms of the diverse constants

$$
\begin{aligned}
\beta_{0}{ }^{2} & =(5 \hbar) /\left[2\left(B_{2} C_{2}\right)^{1 / 2}\right] \\
& =5 E_{2} / 2 C_{2} .
\end{aligned}
$$

It is connected with the $E_{2}$ transition probability of the low-energy spectrum by the relation

$$
B\left(E_{2}\right)=\left[(3 / 4 \pi) Z R_{0}^{2}\right]^{2} \beta_{0}{ }^{2} .
$$

The eigenstates of (18) are $\left|N_{1}, l_{1} ; N_{2}, v_{2}, l_{2} ; I, M\right\rangle$. The quantum numbers $N_{1}, l_{1}$ and $N_{2}, l_{2}$ are the number of phonons and the angular momentum of the dipole and the quadrupole states, respectively, $v_{2}$ is the seniority of the quadrupole states, and $I$ and $M$ are the total angular momentum and its $z$ component.

The interaction $H_{\text {dip quad }}$ is too strong to be treated by perturbation methods. Therefore one has to diagonalize the interaction in the basis of the solutions of $H^{0}$. However the admixture of the 3-phonon dipole state to the 1-phonon state, the usual giant dipole state, still can be treated by perturbation methods since the dipole energy $\hbar \omega_{1}$ is much larger than both the interaction energy and the quadrupole energy $\hbar \omega_{2}$. We therefore neglect in $H_{\text {dip quad }}$ all the terms which do not commute with $\left[d^{\dagger} \times d\right]^{[0]}$ correcting later for the error thus introduced. Denoting the truncated interaction Hamiltonian by $H_{\text {dip quad }}^{\prime}$ we have

$$
\begin{aligned}
H_{\text {dip quad }}^{\prime}= & H_{1}{ }^{\prime}+H_{20}{ }^{\prime}+H_{22}{ }^{\prime} \\
= & -0.16 E_{1} \beta_{0}\left\{\sum _ { \mu \nu } ( - 1 ) ^ { \mu } \left(\left.112\right|_{\left.\nu \mu-\nu \mu)\left[(-1)^{\mu-\nu} d_{\nu}{ }^{\dagger} d_{\nu-\mu}+(-1)^{\nu} d_{-\nu} d_{\mu-\nu}{ }^{\dagger}\right]\left[\beta_{-\mu^{\prime}}{ }^{\dagger}+(-1)^{\mu} \beta_{\mu}\right]\right\}}\right.\right. \\
& -0.05 E_{1} \beta_{0}\left\{\sum_{l=0,2} q_{l} \sum_{\mu \nu \mu^{\prime} \nu^{\prime} M}(-1)^{M}(22 l \mid \mu \nu M)\left(11 l \mid \mu^{\prime} \nu^{\prime}-M\right)\right. \\
& \left.\times\left[(-1)^{\nu^{\prime}} d_{\mu^{\prime}}{ }^{\dagger} d_{-\nu^{\prime}}+(-1)^{\mu^{\prime}} d_{-\mu^{\prime}} d_{\nu^{\prime}}{ }^{\dagger}\right]\left[\beta_{\mu}{ }^{\dagger} \beta_{\nu}^{\dagger}+(-1)^{\nu} \beta_{-\nu} \beta_{\mu}{ }^{\dagger}+(-1)^{\mu} \beta_{\nu}^{\dagger} \beta_{-\mu}+(-1)^{M} \beta_{-\mu} \beta_{-\nu}\right]\right\} ; \\
& q_{0}=1.58, \quad q_{2}=0.94 .
\end{aligned}
$$

The interaction Hamiltonian, $H^{\prime}{ }_{\text {dip quad, }}$ has both diagonal and nondiagonal elements. The diagonal elements of $H_{20}{ }^{\prime}$ have the following effect. After integrating over the dipole oscillations they have the same form as the potential term in $H_{\text {quad }}$. This means that the giant dipole resonances contribute to the restoring force of the surface vibrations. Therefore the $C_{2}$ value, usually taken from the experiment, has to be renormalized. For the case of no dipole oscillation present, $N_{1}=0$, there holds

$$
C_{2}{ }^{\exp }=C_{2}{ }^{0}+0.547 \hbar \omega_{1} .
$$




\begin{tabular}{|c|c|c|c|c|c|c|c|}
\hline $\mathrm{N}_{2}$ & & 0 & 2 & 3 & 4 & 5 & 6 \\
\hline & $v$ & 0 & \begin{tabular}{l|l}
0 & 2 \\
\end{tabular} & \begin{tabular}{|l|l|}
1 & 3 \\
\end{tabular} & \begin{tabular}{|l|l|l|}
0 & 2 & 4 \\
\end{tabular} & \begin{tabular}{|l|l|l|} 
& 3 & 5 \\
\end{tabular} & \begin{tabular}{|l|l|l|l|}
0 & 2 & 4 & 6 \\
\end{tabular} \\
\hline 0 & 0 - 1 & \begin{tabular}{l|l}
$H_{0}$ & $K_{1}$ \\
\end{tabular} & $K_{20} K_{22}$ & & & & \\
\hline $\mathrm{T}$ & 11 & $\begin{array}{l}K_{1} \\
H_{C}\end{array}$ & $\begin{array}{ll}K_{1} \\
\end{array}$ & $\mathrm{~K}_{20} \mathrm{~K}_{22} \mathrm{~W}$ & & & \\
\hline 2 & \begin{tabular}{|l|l}
0 & $r$ \\
2 & \\
\end{tabular} & & $\begin{array}{c}\mathrm{H}_{0} \mathrm{~K}_{20} \mathrm{~W} \\
\mathrm{~K}_{22}\end{array}$ & $k_{1}$ & $\mathrm{~K}_{20} \mathrm{~K}_{22} \mathrm{~W}$ & & \\
\hline 3 & $\frac{1}{2}$ & $\mid \begin{array}{l}k_{2} \\
k_{2} \\
w\end{array}$ & $k_{1}$ & $\begin{array}{c}\mathrm{H}_{0} \mathrm{~K}_{20 \mathrm{O}} \\
\mathrm{K}_{22}\end{array}$ & $k_{1}$ & $k_{20} k_{22} w$ & \\
\hline 4 & $\frac{0}{2}$ & & $\begin{array}{l}K_{20} \\
K_{22} \\
w\end{array}$ & $k_{1}$ & $\begin{array}{cc}H_{0} & K_{20} W \\
& K_{22}\end{array}$ & $k_{1}$ & $K_{20} K_{22} W$ \\
\hline 5 & \begin{tabular}{|l|}
1 \\
2 \\
5 \\
\end{tabular} & & & $\begin{array}{l}\mathrm{K}_{20} \\
\mathrm{~K}_{22} \\
\mathrm{~W}\end{array}$ & $k_{1}$ & $\mid \begin{array}{cc}H_{0} & K_{20} W \\
& K_{22}\end{array}$ & $k_{1}$ \\
\hline 6 & $\begin{array}{l}\frac{0}{2} \\
4 \\
6\end{array}$ & & & & $\begin{array}{l}K_{20} \\
K_{22} \\
w\end{array}$ & $k_{1}$ & $\begin{array}{ll}H_{0} \quad K_{20} W \\
\\
K_{22}\end{array}$ \\
\hline
\end{tabular}

FIG. 1. A schematic picture of the energy matrix. The boxes contain the nonvanishing matrix elements. The contributions of the different parts of the Hamiltonian, Eq. (14), are indicated by the corresponding coupling constants $\left[H_{0}\right.$ and $W$ refer to the unperturbed Hamiltonian, Eq. (15), and the perturbation, Eq. (27), respectively].

$\mathrm{C}_{2}{ }^{0}$ is the renormalized potential constant. For the $1^{-}$ states, where one dipole quantum is excited, one obtains

$$
C_{2}{ }^{\text {eff }}=C_{2}{ }^{\text {exp }}+0.368 \hbar \omega_{1} \text {. }
$$

This means that the restoring force for the surface vibrations is stronger when a giant resonance photon is excited than in the ground-state band. This renormalization effect is for the different nuclei between $5 \%$ and $25 \%$.

Furthermore, the interaction Hamiltonian $H_{\text {dip quad }}^{\prime}$ provides nonvanishing off-diagonal elements. They connect states with different surface quanta and different seniorities. $H_{1}{ }^{\prime}$ admixes states whose number of quadrupole phonons $N_{2}$ and whose seniority $v$ differ by $1, H_{20}{ }^{\prime}$ and $H_{22}{ }^{\prime}$ change $N_{2}$ by 2 and $v$ by 0 or 2 , respectively. A schematic picture of the energy matrix is given in Fig. 1. The elements of the different parts of the interaction Hamiltonian are indicated by the corresponding coupling constants. The matrix elements of $H^{\prime}{ }_{\text {dip quad }}$ recently have been evaluated. ${ }^{10}$ Even though these calculations were somewhat involved, the algebraic expression for the elements are relatively simple.

All the contributions which do not conserve the number of dipole phonons have been neglected in the interaction Hamiltonian $H^{\prime}{ }_{\text {dip quad, }}$ i.e., the terms of the form $\left[d^{\dagger} d^{\dagger} \beta^{\dagger}\right]^{[0]}$ or $[d d \beta]^{[0]}$ which admix the threephonon states to the one-dipole phonon state have been omitted. This part, $W$, of the Hamiltonian can be separated into two terms, $W_{1}$ and $W_{2}$,

$$
W=H-H_{0}-H_{\text {dip quad }}^{\prime}=W_{1}+W_{2}
$$

which arise from $H_{1}$ and $H_{20}+H_{22}$, respectively. We

${ }^{10}$ H. J. Weber, M. G. Huber, and W. Greiner, Z. Physik 192, 182 (1966). give a rough estimate of the energy shift produced by $W_{1}$ :

$$
\begin{aligned}
W_{1}=- & 0.356 h \omega_{1} \beta_{0}\left\{\left[\left[d^{\dagger} \times d^{\dagger}\right]^{[2]} \times \beta^{\dagger}\right]^{[0]}\right. \\
+\left[\left[d^{\dagger} \times d^{\dagger}\right]^{[2]} \times \beta\right]^{[0]} & +\left[[d \times d]^{[2]} \times \beta^{\dagger}\right]^{[0]} \\
& \left.+\left[[d \times d]^{[2]} \times \beta\right]^{[0]}\right\} .
\end{aligned}
$$

As stated earlier, we will treat this interaction by perturbation methods. Then one obtains for the energy shift

$$
\begin{aligned}
\Delta E & =\sum_{f} \frac{\left|\left\langle i\left|W_{1}\right| f\right\rangle\right|^{2}}{E_{i}-E_{f}} \\
& \approx-\frac{1}{2 \hbar \omega_{1}} \sum_{f}\left|\left\langle i\left|W_{1}\right| f\right\rangle\right|^{2} \\
& \approx-\frac{1}{2 \hbar \omega_{1}}\left\langle i\left|W_{1}{ }^{2}\right| i\right\rangle,
\end{aligned}
$$

using the approximation $\left|E_{i}-E_{f}\right| \approx 2 \hbar \omega_{1}$. A rough estimate leads to $|\Delta E|<0.4 \mathrm{MeV}$. This justifies $a$ posteriori the application of perturbation methods as well as the neglect of the interaction $W_{2}$, which is smaller than the contribution of $W_{1}$ by a factor of about $\beta_{0}^{2}$, i.e., 0.1 to 0.01 . Explicit formulas for the contribution of $W_{1}$ to the different matrix elements are given in Ref. 10.

In Fig. 1 the matrix elements which are affected by $W_{1}$ are schematically indicated by $W$.

\section{THE DIPOLE OPERATOR}

The dipole operator is defined by

$$
D_{\nu}=\left(\frac{4 \pi}{3}\right)^{1 / 2} \int_{\mathrm{vol}} \rho_{p} r Y_{1 \nu} d V .
$$

Here the integration has to be performed over the whole nuclear volume. Using the relations (5a), (8) and (2), its general form is easily seen to be $\mathrm{e}^{11}$

$$
D^{[1]}=D_{0}\left\{\alpha^{[1]}+D_{1}\left[\alpha^{[1]} \times \alpha^{[2]}\right]^{[1]}\right\},
$$

neglecting higher-order terms. The constants $D_{0}$ and $D_{1}$ can be calculated straightforwardly in the adiabatic approximation. As already stated, this means that the nucleus actually is deformed as far as the giant dipole resonances are concerned. In the intrinsic coordinate system the dipole resonance generally splits into three nondegenerate modes. Their energies are determined by the relation (12). Introducing a spherical rather than a Cartesian frame of reference for the representation of the wave functions, the matrix elements of the dipole operator (28) can be evaluated in the intrinsic coordinate system. Similarly, the matrix elements of the dipole operator (29) can also be com-

${ }^{11} \mathrm{H}$. Arenhoevel, dissertation, University of Frankfurt, 1965 (to be published). 
puted after transformation into the intrinsic system. Comparing both these expressions the constants $D_{0}$ and $D_{1}$ in Eq. (29) are found to be

$$
\begin{aligned}
& D_{0}=(0.478 h)\left[6 N Z /\left(\pi A m^{*} h \omega_{1}\right)\right]^{1 / 2}, \\
& D_{1}=-0.247 \beta_{0} .
\end{aligned}
$$

Here $m^{*}$ is the effective mass of the nucleon. It should be pointed out that the dipole operator, Eq. (29), takes into account only transitions into the lowest dipole mode. ${ }^{12}$ So the corresponding integrated cross section covers only $86 \%$ of the dipole sum rule. ${ }^{9}$ It is common use to interpret the effective mass $m^{*}$ in terms of an enhancement factor ${ }^{13}(1+\alpha)$, which in our case is defined by

$$
m^{*}=0.86 m /(1+\alpha) \text {. }
$$

\section{THE PHOTON-ABSORPTION CROSS SECTION}

All the states calculated in Sec. II are eigenstates of the Hamiltonian (14). They will be broadened by the coupling of the other degrees of freedom. The most important damping mechanism is the thermalization of the energy. ${ }^{14}$ Here we shall restrict ourselves to a phenomenological description of this process by introducing a total width of the states. The widths of the different states, $\Gamma_{k}$, may be different. The photonabsorption cross section then is given by

$$
\sigma(E)=\sum_{k} \frac{\sigma_{k}}{\left[\left(E^{2}-E_{k}^{2}\right) / E \Gamma_{k}\right]^{2}+1},
$$

where $\sigma_{k}$ is given by

$$
\sigma_{k}=8 \pi\left(e^{2} / \hbar c\right)\left|\left\langle\Phi_{0}|D| \Psi_{k}\right\rangle\right|^{2}\left(E_{k} / \Gamma_{k}\right) .
$$

Here $\Phi_{0}$ represents the ground state. Corresponding to the structure of the dipole operator in (28), the matrix element has the following form:

$$
\begin{aligned}
\left|\left\langle\Phi_{0}|D| \Psi_{k}\right\rangle\right|^{2} & =D_{0}^{2} \varphi_{k}^{2}, \\
\varphi_{k} & =p_{k}+D_{1} q_{k} .
\end{aligned}
$$

Here the admixture coefficients of the components $|1,1 ; 0,0,0 ; 1 M\rangle$ and $|1,1 ; 1,1,2 ; 1 M\rangle$ of the wave function are given by $p_{k}$ and $q_{k}$, respectively.

Inserting $\sigma_{k}$ in (32), one finally obtains for the photon-absorption cross section

$$
\begin{aligned}
\sigma(E)=0.038(1+\alpha)\left(\frac{N Z}{A}\right) & \sum_{k} \frac{E_{k} \varphi_{k}^{2}}{E_{1} \Gamma_{k}} \\
& \times \frac{1}{\left[\left(E^{2}-E_{k}^{2}\right) / E \Gamma_{k}\right]^{2}+1} .
\end{aligned}
$$

(All energies in $\mathrm{MeV}, \sigma$ in $10^{-24} \mathrm{~cm}^{2}$.)

\footnotetext{
${ }^{12}$ We thank E. G. Fuller for clarifying discussions of this point. ${ }^{13}$ J. S. Levinger, Nuclear Photodisintegration (Oxford University Press, New York, 1960).

${ }^{14}$ M. Danos and W. Greiner, Phys. Rev. 138, B876 (1965).
}

\section{COMPARISON WITH EXPERIMENTS}

The photon-absorption cross section is given by

$\sigma^{\mathrm{abs}}=\sigma(\gamma, n)+\sigma(\gamma, p)+\sigma(\gamma, p n)+\sigma(\gamma, 2 p)+\sigma(\gamma, 2 n)$.

For energetic reasons the emission of more than two particles is excluded in most nuclei (see Fig. 2). For medium and heavy elements the emission of protons is strongly inhibited by the Coulomb barrier, and except for the $\mathrm{Ni}$ isotopes their contribution to the total cross section is less than $10 \%{ }^{15}$ In the following discussion we therefore will neglect the contribution of the processes involving proton emission. Then we obtain

$$
\sigma^{\mathrm{abs}}=\sigma(\gamma, n)+\sigma(\gamma, 2 n) .
$$

In addition to the resonating process direct continuum transitions also contribute to the absorption. They lead to fast-neutron emission. Thus we have

$$
\sigma(\gamma, n)=\sigma^{\text {coll }}(\gamma, n)+\sigma^{\text {direct }}(\gamma, n) .
$$

In order to obtain the cross section for the excitation of the collective dipole states one, therefore, has to subtract this nonresonating contribution:

$$
\sigma^{\text {dip }}=\sigma^{\text {coll }}(\gamma, n)+\sigma(\gamma, 2 n)=\sigma^{\text {abs }}-\sigma^{\text {direct }}(\gamma, n) .
$$

Unfortunately, very little is knwon about $\sigma^{\text {direct }}(\gamma, n)$. From several experiments ${ }^{16}$ one can conclude that their contribution to the integrated cross section is less than $15 \%$. Furthermore, we assume a smooth energy dependence. The uncertainties introduced by this correction are indicated in the figures by the boxes.

The measured neutron-yield cross sections

$$
\sigma^{\exp }=\sigma^{\operatorname{coll}}(\gamma, n)+2 \sigma(\gamma, 2 n)+\sigma^{\operatorname{direct}}(\gamma, n)
$$

have to be corrected for the neutron multiplicity. This can be done using the statistical neutron-evaporation

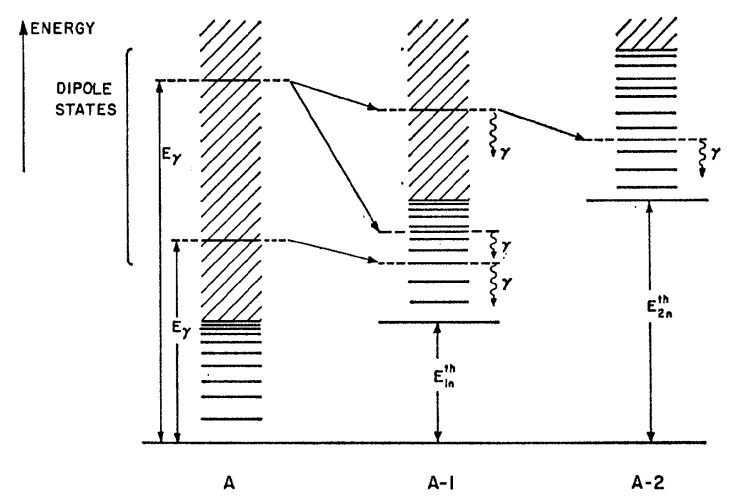

Fig. 2. Decay scheme of the giant resonance states. The hatched area represents the continuum states. Each arrow symbolizes the emission of a particle (proton or neutron). $E_{\gamma}$ is the energy of the absorbed $\gamma$ quantum.

${ }^{15}$ E. Hayward, Photonuclear Reactions, Scottish Universities's Summer School, 1964 (unpublished).

${ }^{16} \mathrm{G}$. Mutchler, Ph.D. thesis, Massachusetts Institute of Technology, 1965 (unpublished). 
theory. The multiplicity correction factor $F(E)$ is defined by

$$
\begin{aligned}
\sigma^{\mathrm{coll}} & =\sigma^{\exp }-\sigma^{\text {direct }}(\gamma, n)-\sigma(\gamma, 2 n) \\
& =\left\{\sigma^{\exp }-\sigma^{\text {direct }}(\gamma, n)\right\} F(E) .
\end{aligned}
$$

Then we have ${ }^{17}$

$$
\begin{array}{rlrl}
F(E) & =1 /\left[2-(1+X) e^{-X}\right] & \text { for } & E>E_{2 n}^{\text {th }}, \\
& =1 & \text { for } E<E_{2 n}^{\text {th }}, \\
X & =\left(E-E_{2 n^{\text {th }}}\right) / \theta . & &
\end{array}
$$

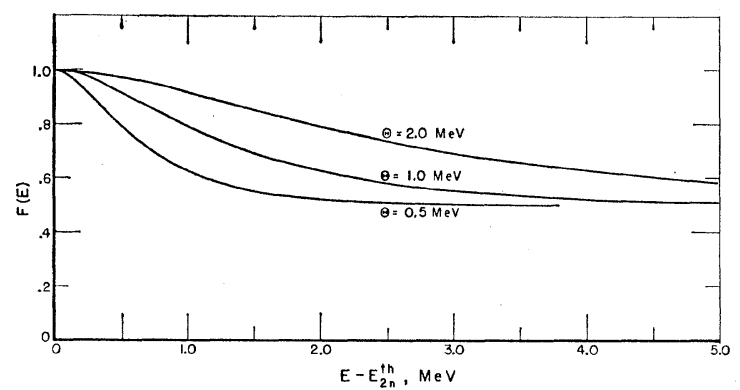

FIG. 3. Neutron multiplicity correction. $F(E)$ is the factor which relates the experimental to the total $(\gamma, n)$ cross section.

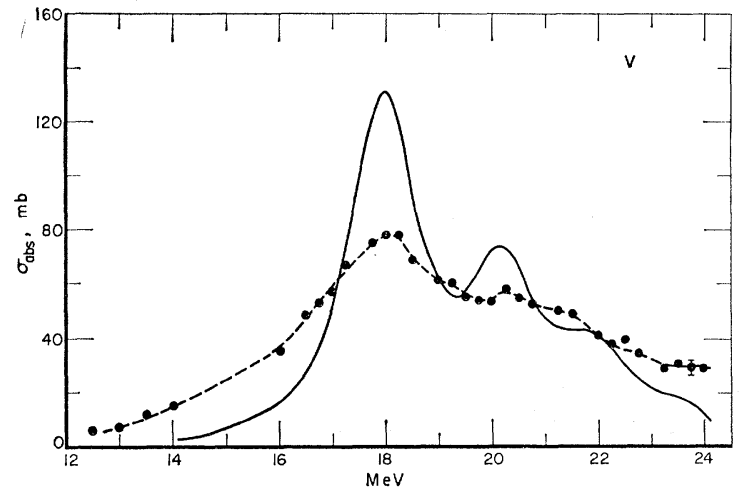

Fig. 4. Photon-absorption cross section of $\mathrm{V}^{51}$. The dashed line represents the experimental points (Ref. 18), the solid line the calculations.

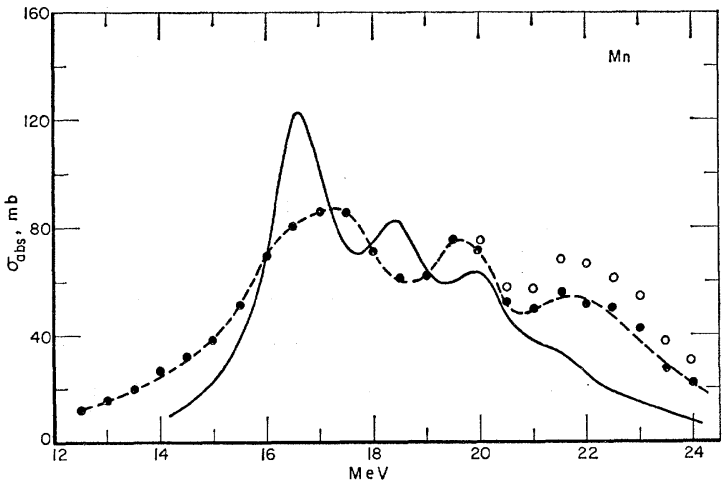

Fig. 5. Cross section of $\mathrm{Mn}^{55}$. The dashed line represents the experimental points (Ref. 19), the solid line the calculations.

${ }_{17}$ J. M. Blatt and V. F. Weisskopf, Theoretical Nuclear Physics (John Wiley \& Sons, Inc., New York, 1952).
$E_{2 n}{ }^{\text {th }}$ is the threshold energy for the two-neutron emission. The temperature $\theta$ was chosen to be $1 \mathrm{MeV}$. In Fig. 3 this correction factor has been plotted for several temperatures; as can be seen, small uncertainties in $\theta$ have no appreciable effect on the corrections. Up to now only a few $(\gamma, n)$ cross sections in the region of medium-heavy nuclei have been measured. In Figs. 4 through 10 the theory developed in the preceding sections is compared with the presently available experimental data from $\mathrm{V}^{51}$ through $\mathrm{As}^{75} \cdot{ }^{18-21}$ With the exception of $V^{51}$ the experiments give the total neutron-yield cross section. The parameters used for the conversion to the giant resonance absorption cross section by means of (41) are collected in Table I.

In the calculations of the theoretical absorption cross section, parameters obtained from the neighboring even-even nuclei were used. They are listed in Table II. As an example, the principal dipole states of $\operatorname{Pr}^{141}$ are listed in Table III. The surface phonon states are classified by $N_{2}$ and $v$ (see Fig. 1). The calculations have been performed using all states up to 8 phonons, i.e., a $25 \times 25$ matrix was diagonalized. Only the

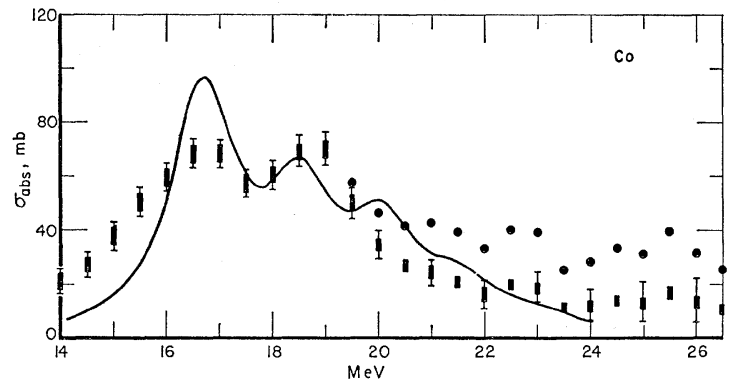

Fig. 6. Cross section of natural cobalt. The experimental points are from Ref. 20. The solid line represents the calculated cross section. Here and in the following figures the uncertainties due to the direct emission of neutrons are indicated by the boxes.

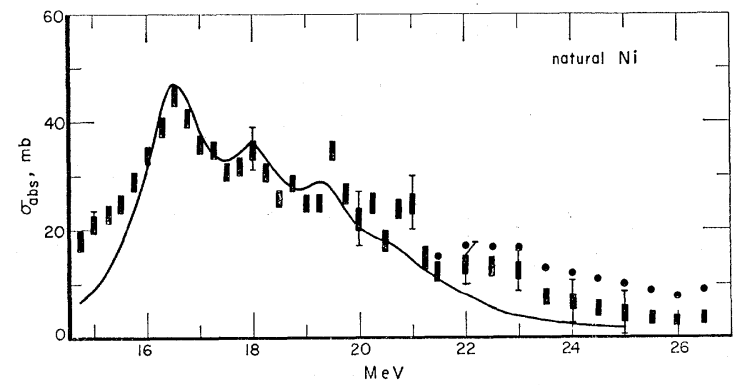

Fig. 7. Experimental (Ref. 20) and calculated (solid line) cross section of natural nickel.

${ }^{18}$ S. C. Fultz, R. L. Bramblett, J. T. Caldwell, N. E. Hansen, and C. P. Jupiter, Phys. Rev. 128, 2345 (1962).

${ }^{19}$ P. A. Flournoy, R. S. Tickle, and W. D. Whitehead, Phys. Rev. 120, 1424 (1960).

${ }^{20}$ G. Baciu, G. C. Bonazzola, B. Minetti, C. Molino, L. Pasqualini, and G. Piragino, Nucl. Phys. 67, 178 (1965).

${ }^{21}$ D. S. Fielder, J. LeTourneux, K. Min, and W. D. Whitehead, Phys. Rev. Letters 15, 33 (1965). 
states with up to two phonons are listed here. In contrast to the deformed nuclei it seems that here the width of the giant resonance states is a very slow function of the energy. Therefore we assumed in our calculations the width to be the same for all the different states. Furthermore we have adjusted the integrated cross section. The effective mass parameters $m^{*} / m$ (compare Table II), i.e., the exchange-force corrections, are generally not in contradiction with the results of Levinger ${ }^{13}$ considering the fact that they cannot be well determined from the present experiments because of the uncertainties of the absolute value of the neutronyield cross sections, the contribution of the direct neutrons, and of the emission of protons.

As can be seen from the figures, the experimental cross sections have a marked structure. The calculated

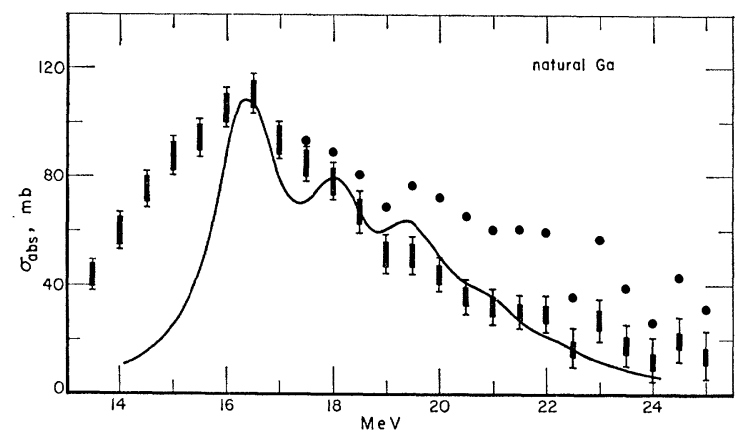

Fig. 8. Experimental (Ref. 20) and calculated (solid line) cross section of natural gallium.

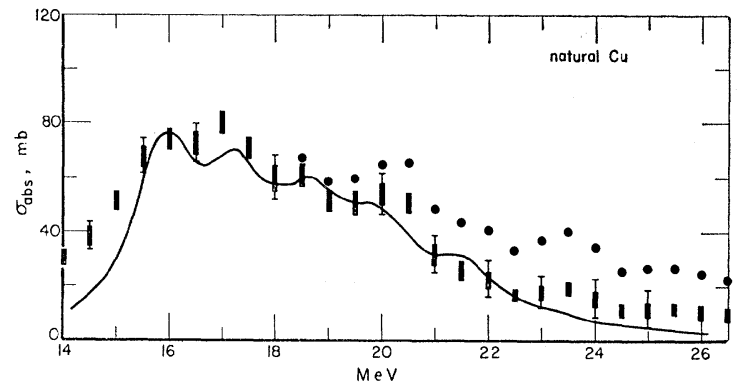

FIG. 9. Experimental (Ref. 20) and calculated (solid line) cross section of natural copper.

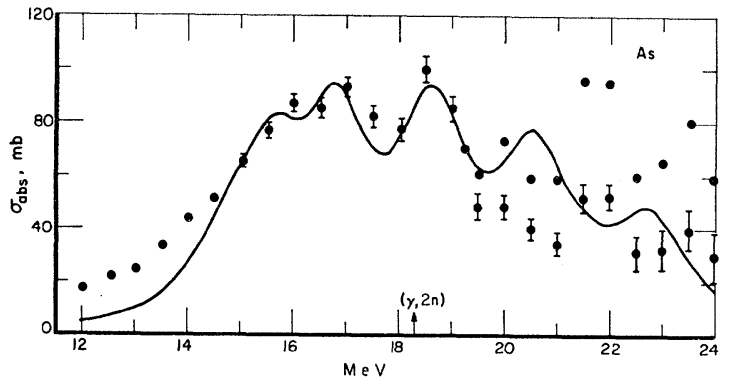

Fig. 10. Experimental (Ref. 21) and calculated (solid line) cross section of $\mathrm{As}^{75}$.
TABLE I. Relative abundances and threshold energies for the $2 n$ emission of the nuclei studied in detail.

\begin{tabular}{ccc}
\hline \hline Nucleus & $\begin{array}{c}\text { Relative } \\
\text { abundances }\end{array}$ & $E_{2 n^{\text {th }}}(\mathrm{MeV})$ \\
\hline $\mathrm{Mn}^{55}$ & 100.0 & 19.2 \\
$\mathrm{Co}^{59}$ & 100.0 & 19.0 \\
$\mathrm{Ni}^{58}$ & 67.9 & 22.5 \\
$\mathrm{Ni}^{60}$ & 26.2 & 20.4 \\
$\mathrm{Co}^{63}$ & 69.1 & 19.7 \\
$\mathrm{Co}^{65}$ & 30.9 & 17.8 \\
$\mathrm{Ga}^{69}$ & 60.4 & 16.6 \\
$\mathrm{Ga}^{71}$ & 39.6 & 17.0 \\
$\mathrm{As}^{75}$ & 100.0 & 18.3 \\
\hline
\end{tabular}

TABLE II. Parameters used in the calculation of the photon-absorption cross sections.

\begin{tabular}{lccccc}
\hline \hline \multicolumn{1}{c}{ Nucleus } & $\beta_{0}$ & $\begin{array}{c}E_{2} \\
(\mathrm{MeV})\end{array}$ & $\begin{array}{c}E_{1} \\
(\mathrm{MeV})\end{array}$ & \multicolumn{1}{c}{$\alpha$} & $\begin{array}{c}\Gamma \\
(\mathrm{MeV})\end{array}$ \\
\hline${ }_{23} \mathrm{~V}^{51}$ & 0.20 & 1.60 & 19.3 & 0.0 & 1.5 \\
${ }_{25} \mathrm{Mn}^{55}$ & 0.22 & 1.30 & 18.2 & 0.0 & 1.5 \\
${ }_{27} \mathrm{Co}^{59}$ & 0.22 & 1.30 & 18.3 & -0.3 & 1.5 \\
Natural Ni & 0.20 & 1.10 & 18.0 & -0.6 & 1.5 \\
Natural $\mathrm{Cu}$ & 0.25 & 1.00 & 18.1 & -0.3 & 1.5 \\
Natural Ga & 0.22 & 1.20 & 18.0 & -0.3 & 1.5 \\
${ }_{33} \mathrm{As}^{75}$ & 0.31 & 0.64 & 18.5 & 0.0 & 1.5 \\
${ }_{59} \mathrm{Pr}^{141}$ & 0.11 & 1.40 & 16.0 & -0.1 & 1.5 \\
${ }_{82} \mathrm{~Pb}^{208}$ & 0.15 & 4.20 & 14.0 & 0.0 & 1.5 \\
\hline \hline
\end{tabular}

TABLE III. Energies, dipole strengths, and wave functions of the principal dipole states of $\operatorname{Pr}^{141}$. The parameters used in the calculations are listed in Table II.

\begin{tabular}{ccrrrr}
\hline \hline & & \multicolumn{5}{c}{ Amplitudes } \\
$E(\mathrm{MeV})$ & $\varphi^{2}$ & $N_{2}=0$ & 1 & 2 & 2 \\
& $v=0$ & 1 & 0 & \multicolumn{1}{c}{2} \\
\hline 15.647 & 0.846 & 0.91 & -0.41 & 0.06 & 0.07 \\
17.526 & 0.142 & 0.40 & 0.78 & -0.30 & -0.36 \\
18.868 & 0.003 & 0.07 & 0.24 & 0.83 & -0.24 \\
19.154 & 0.009 & 0.10 & 0.39 & 0.01 & 0.79 \\
& & & & & \\
\hline
\end{tabular}

cross sections are in fairly good agreement with the experimental data. They reproduce the number, the energies, and even the dipole strengths of the absorption peaks quite well. It is striking that even some of the finer details are reproduced by the theoretical calculation without the introduction of additional parameters. Nevertheless, there still exist discrepancies between theory and experiment. This is to be expected since several factors, which should have an influence on the giant resonance, have been neglected in this treatment. One of them is the unpaired particle. Others are the diverse nonharmonic terms, i.e., the terms represented by the dots in Eq. (11). Finally, the central assumption of this treatment, viz., that, in the shellmodel language, the interaction between the $1^{-}$oneparticle-one-hole configurations results in one collective dipole state definitely is not completely fulfilled in the medium-heavy nuclei. Some of the dipole strength may still be left at the positions of the unperturbed energies. 


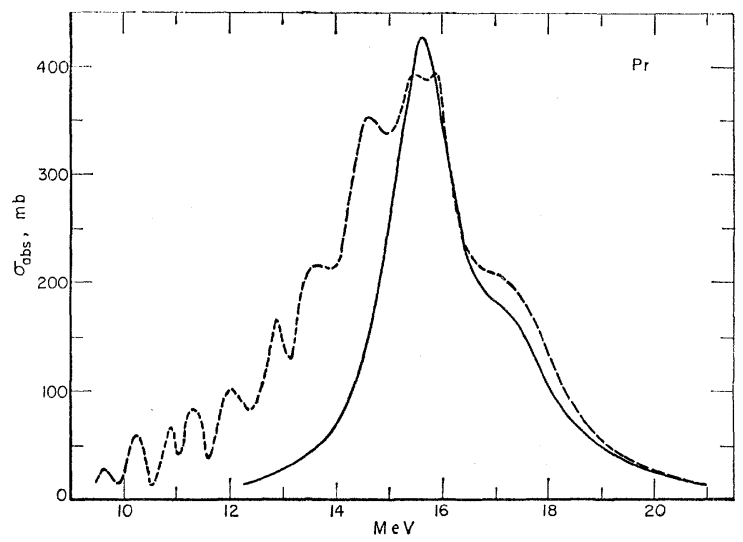

Fig. 11. Experimental (Ref. 22) (dashed line) and calculated (solid line) cross section of $\operatorname{Pr}^{141}$.

This effect is expected to become the more important the more the single-particle aspects prevail, viz., in the shell-model nuclei. Two examples are given in Figs. 11 and $12 . .^{22-24}$ There both the cross sections of ${ }_{59} \operatorname{Pr}_{82}{ }^{141}$ and of ${ }_{82} \mathrm{~Pb}_{126}{ }^{208}$ clearly show a pronounced structure on the leading edge of the giant resonance peak which apparently is not reproduced by the present treatment. This fact will be discussed in more detail in Sec. VIII. There also may be indications of the existence of the giant quadrupole resonances on the high-energy side of the cross section. ${ }^{25}$ Despite these discrepancies the comparison between the experimental and the calculated cross sections seems to indicate that the present theoretical treatment is able to describe the essential properties of the giant resonance spectrum in spherical nuclei.

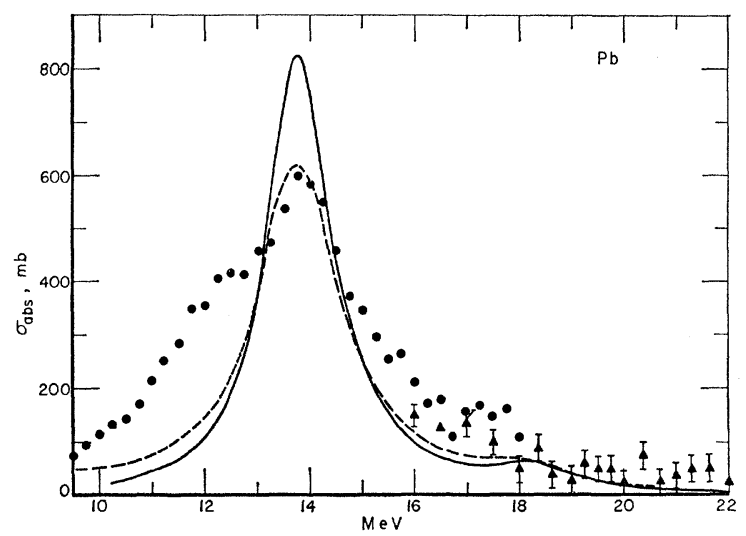

FIg. 12. Experimental (Ref. 23) and calculated (solid line) cross section of $\mathrm{Pb}^{208}$ (see Ref. 24).

22 B. C. Cook, D. R. Hutchinson, R. C. Waring, J. N. Bradford, R. G. Johnson, and J. E. Griffin, Phys. Rev. 143, 730 (1966)

${ }_{23}$ E. G. Fuller and E. Hayward, Nucl. Phys. 33, 431 (1962).

${ }_{24}^{24}$ The existence of a collective $2^{+}$level in $\mathrm{Pb}^{208}$ recently has been reported by J. Alster, Phys. Rev. 141, 1138 (1966).

${ }^{25}$ R. Ligensa, W. Greiner, and M. Danos, Phys. Rev. Letters 16, 363 (1966).

\section{PREDICTIONS}

In this section we shall describe the way in which the photon-absorption cross section depends on the different nuclear parameters and we shall give some examples as a guide to the planning of photonuclear experiments. We shall give only the dipole strengths $\varphi_{k}^{2}$, Eq. (34), to the different dipole states, because no numerically accurate theory exists at the present concerning the widths. The photon-absorption cross section can be obtained from the strengths by assuming the total widths of the diverse states.

The unperturbed giant resonance oscillations are determined by the constants, $C_{1}$ and $B_{1}$, in the Hamiltonian $H_{\text {dip }}$, Eq. (10). In the hydrodynamic model their energy is given by the relation

$$
E_{1}=80 A^{-1 / 3} \mathrm{MeV} \text {. }
$$

This formula is experimentally well proven for $A>100$. For lighter nuclei one observes deviations up to $10 \%$

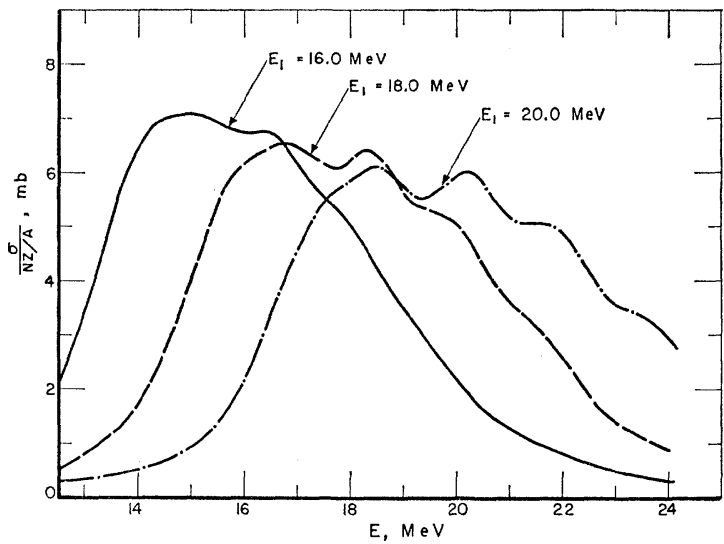

FIG. 13. Dipole-absorption cross section for different energies of the dipole phonons $\left(E_{2}=0.6 \mathrm{MeV}, \beta_{0}=0.25, N_{\text {phonon }}=8, \Gamma=2.0\right.$ $\mathrm{MeV})$.

from (43). $\cdot^{15}$ From the present available experimental data one cannot determine a systematical trend in these deviations. Furthermore, no theoretical refinement of the energy formula of the hydrodynamic model exists which takes into account the different possible modifications affecting the relation (43). Therefore one has to treat the unperturbed dipole energy $E_{1}$ as a free parameter.

The dependence of the dipole absorption spectrum when only $E_{1}$ is varied is shown in Fig. 13 ; the calculations were carried out assuming a linewidth $\Gamma_{k}=1$ $\mathrm{MeV}$. As can be seen, a variation of $E_{1}$ results mainly in a shift of the spectrum. Therefore it will be sufficient to plot the absorption spectrum only for one value, $E_{1}{ }^{0}$. For the present calculations we assumed $E_{1}{ }^{0}=18.0$ $\mathrm{MeV}$. As can be seen from Fig. 13, a variation of $E_{1}$ produces, in addition to the energy shift, also a slight modification of the spectrum. This results from the dependence of the coupling strengths on $E_{1}$, viz., the 


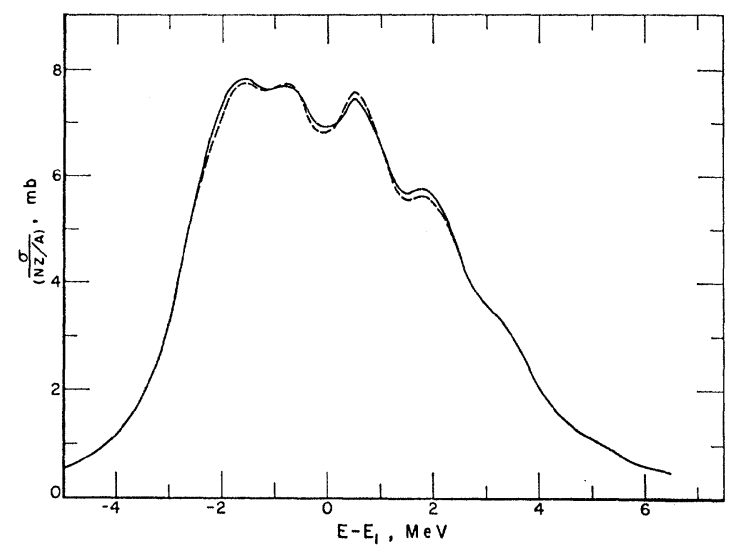

FIG. 14. Cross section for different values of $E_{1}$. The $\beta_{0}$ value has been chosen so that the coupling constants are the same in both cases. (Solid line: $E_{1}=20.0 \mathrm{MeV}, \beta_{0}=0.20$; dashed line: $E_{1}=16.0 \mathrm{MeV}, \beta_{0}=0.25 ; E_{2}=0.6 \mathrm{MeV}, N_{\text {phonon }}=8, \Gamma=1.5$ $\mathrm{MeV})$.

first- and the second-order interaction terms contain $\beta_{0} E_{1}$ and $\beta_{0}^{2} E_{1}$, respectively, as can be seen in Eq. (22). This influence of the dipole energy $E_{1}$ on the spectrum can be canceled out in part by choosing a value for $\beta_{0}$ such that the coupling energy of the first interaction term still remains unchanged. So, if the spectrum is calculated for an energy, say $E_{1}{ }^{0}$, then one has to use $\bar{\beta}_{0}$ given by the relation

$$
\bar{\beta}_{0}=\beta_{0}\left(E_{1} / E_{1}^{0}\right) .
$$

The cross sections for two different values of $E_{1}$, viz., 16 and $20 \mathrm{MeV}$, are plotted in Fig. 14 while the $\beta_{0}$ was chosen to be 0.25 and 0.20 , respectively. As can be seen, the two curves no longer show any noticeable difference in shape after correcting for the energy shift. Therefore it will be sufficient to evaluate the dipole strengths only for one value of $E_{1}$. This means that for an actual nucleus with the dipole energy $E_{1}$ we have to apply the spectrum calculated with $\bar{\beta}_{0}$ rather than that calculated

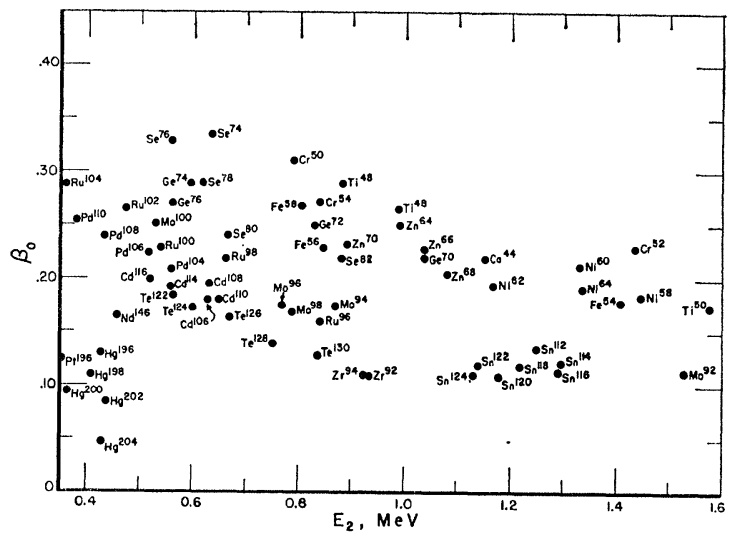

FIG. 15. Energies $E_{2}$ and mean vibrational amplitudes $\beta_{0}$ of some even-even nuclei.

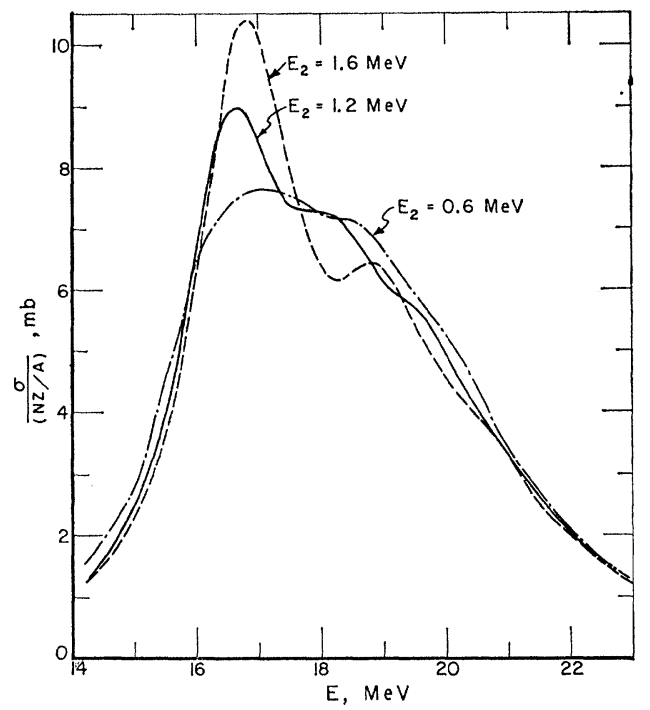

FIG. 16. Calculated dipole-absorption cross section for different values of the quadrupole phonon energy $E_{2}\left(E_{1}=18.0 \mathrm{MeV}\right.$, $\beta_{0}=0.20, N_{\text {phonon }}=8, \Gamma=2.0 \mathrm{MeV}$ ).

with $\beta_{0}$ and to shift the spectrum by the amount $E_{1}-E_{1}^{0}$.

The low-energy properties of the vibrational nuclei are given in the harmonic approximation by the quadrupole phonon energy $E_{2}$, Eq. (19), and the mean vibrational amplitude $\beta_{0}$, Eq. (20), which can be obtained from the experimental $B(E 2)$ values by means of Eq. (21). These two parameters also determine the interaction between the surface vibrations and the dipole oscillations. In Fig. 15 the values of $\beta_{0}$ and $E_{2}$ are plotted for a number of spherical even-even nuclei. Usually the quadrupole energy $E_{2}$ lies between 0.4 and $1.2 \mathrm{MeV}$. In this region the calculated photon-absorption cross section depends only weakly on the exact value of $E_{2}$ (see Fig. 16). We therefore have computed

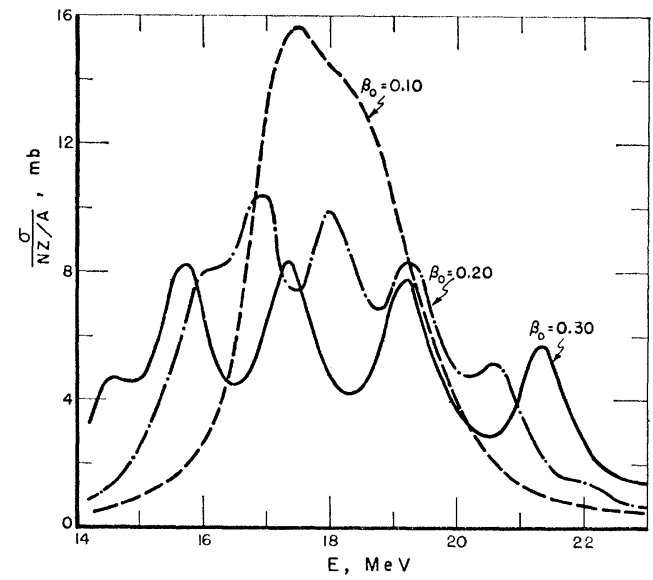

FIG. 17. Calculated dipole-absorption cross section for different values of the vibrational parameter $\beta_{0}\left(E_{1}=18.0 \mathrm{MeV}, E_{2}=0.4\right.$ $\mathrm{MeV}, N_{\text {phonon }}=8, \Gamma=1.0 \mathrm{MeV}$ ). 


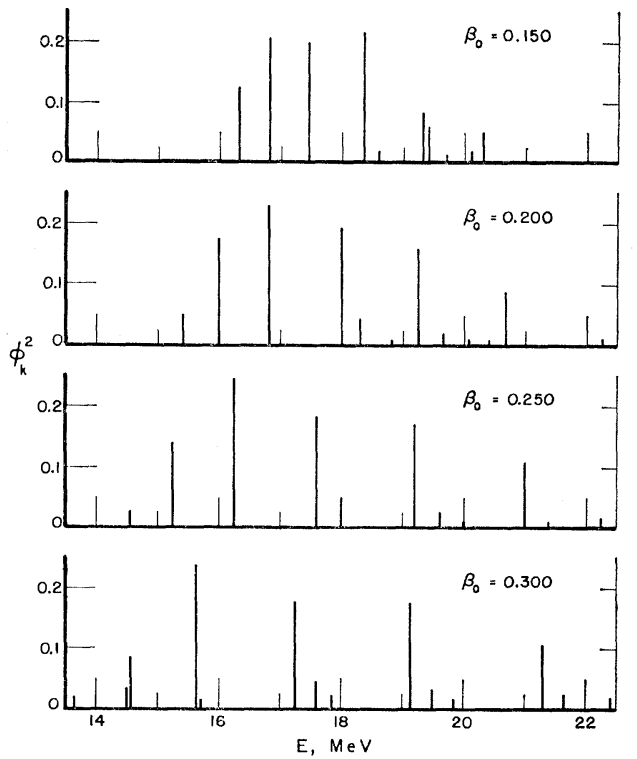

FIG. 18. Dipole strengths $\varphi_{\mathbf{k}}^{2}$ as defined in Eq. (34) for different values of $\beta_{0}\left(E_{1}=18.0 \mathrm{MeV}, E_{2}=0.4 \mathrm{MeV}, N_{\text {phonon }}=8\right)$.
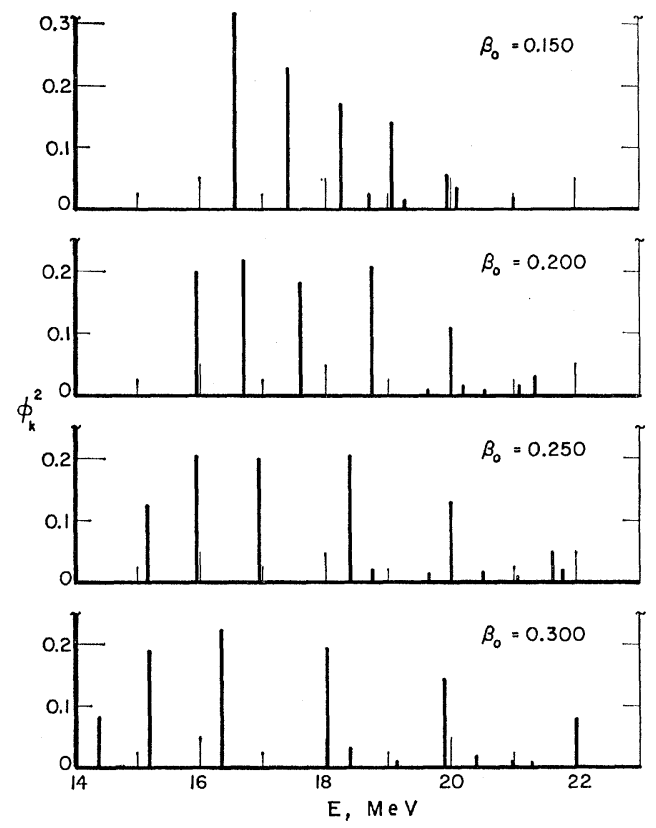

FIG. 19. Dipole strengths $\varphi_{\mathrm{k}}^{2}$ for different values of $\beta_{0}$ $\left(E_{1}=18.0 \mathrm{MeV}, E_{2}=0.6 \mathrm{MeV}, N_{\text {phonon }}=8\right)$.

the dipole strengths for $E_{2}=0.4,0.6,0.9,1.2,1.6 \mathrm{MeV}$ which should suffice for interpolation purposes. Actually the spectrum of the $1^{-}$states is determined mainly by the vibrational amplitude, i.e., $\beta_{0}$. This can be seen in Fig. 17, where the cross sections are plotted for several values of $\beta_{0}$. The widths of the states have been assumed to be $1.0 \mathrm{MeV}$.

The dipole strengths $\varphi_{k}^{2}$ have been plotted in Figs.
18 through 22 as a function of $\beta_{0}$ for different values of $E_{2}$ in the region of physical interest, $\beta_{0}<0.4$. From these figures one easily can evaluate the dipole strengths for an arbitrary spherical nucleus in this mass region. As already stated, the Hamiltonian matrix to be diagonalized is in principle infinite. For practical reasons one has to restrict the number of interacting phonons. How many phonons one actually has to take into account depends on the value of $\beta_{0}$ and the energy $E_{2}$ of the quadrupole phonons. The computed dipole strengths using different numbers of interacting phonons are plotted in Fig. 23. One sees that for the typical values $E_{2}=0.6 \mathrm{MeV}$ and $\beta_{0}=0.2$ it will be sufficient to take six phonons into account. Usually we did the calculations with eight phonons. One then has to treat the interaction between 25 eigenstates of the unperturbed Hamiltonian $H_{0}$.

\section{CONCLUSIONS AND DISCUSSION}

As can be seen from Figs. 4 through 12, the experimental cross sections are reproduced by the theory in a semiquantitative manner. The spreading of the resonance over energy is given quite well by the theory, and also the "collective" structure is qualitatively reproduced, in particular in the stiffer vibrators, e.g., $\mathrm{V}^{51}, \mathrm{Mn}^{55}, \mathrm{~Pb}^{208}$. However, in some cases the rising side of the resonance, i.e., the region below the "main peak," shows structure which is not accounted for by the theory. To understand the reason for the successes and the failure of the theory one has to take a more fundamental point of view, i.e., one has to interpret the theory in terms of the shell model.

Both the giant resonance and the vibrational states are one-particle-one-hole (1-ph) states. More precisely, a one-phonon state is a 1-ph state, a two-phonon state

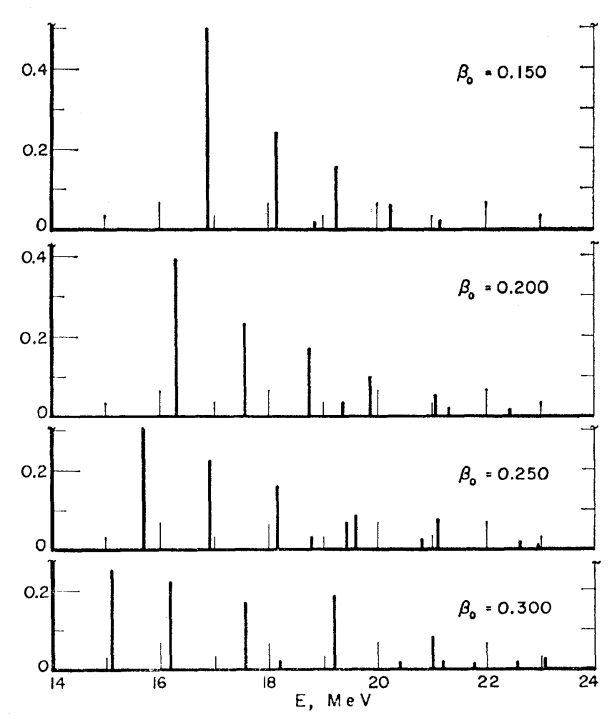

FIG. 20. Dipole strengths $\varphi_{\mathbf{k}}{ }^{2}$ for different values of $\beta_{0}$ $\left(E_{1}=18.0 \mathrm{MeV}, E_{2}=0.9 \mathrm{MeV}, N_{\text {phonon }}=8\right)$. 
is a 2-ph (two-particle-two-hole) state, etc. In the vibrational region of the periodic table the particle states and the hole states contained in the surface phonons and the giant resonance are to a large extent, but not completely, different. It is thus reasonable that these two modes can exist side by side and interact essentially only via the boundary conditions of the giant dipole resonance, i.e., by the tuning of the dipole mode by the surface and by the adiabatic reaction of the dipole mode on the surface. The situation is quite different when many-phonon states of the surface vibrations are considered. A phonon state arises by the diagonalization of the appropriate 1-ph states. In a harmonic description of the surface vibrations a twophonon state is a 2-ph state consisting of two noninteracting 1-ph states. There is no reason to assume that the two particles or the two holes of the two 1-ph states do not interact, and, as a matter of fact, it is frequently impossible to tell which of the particles and holes are the "real" and which are the "crossed" partners. In short, the two 1-ph components of the two-phonon states must be expected to interact strongly. The same arguments, even more forcefully, apply to the many-phonon states.

To include such interactions in a collective description one clearly must add anharmonic terms to the Hamiltonian. The Hamiltonian (1) still does not include such terms; only quadratic terms in $\alpha$ have been considered up till now. Still, it is not clear at all that it would be sufficient to add such anharmonic terms. It very well could be true that the many-phonon states lose completely the phonon character, i.e., they may retain very little of a structure corresponding to the

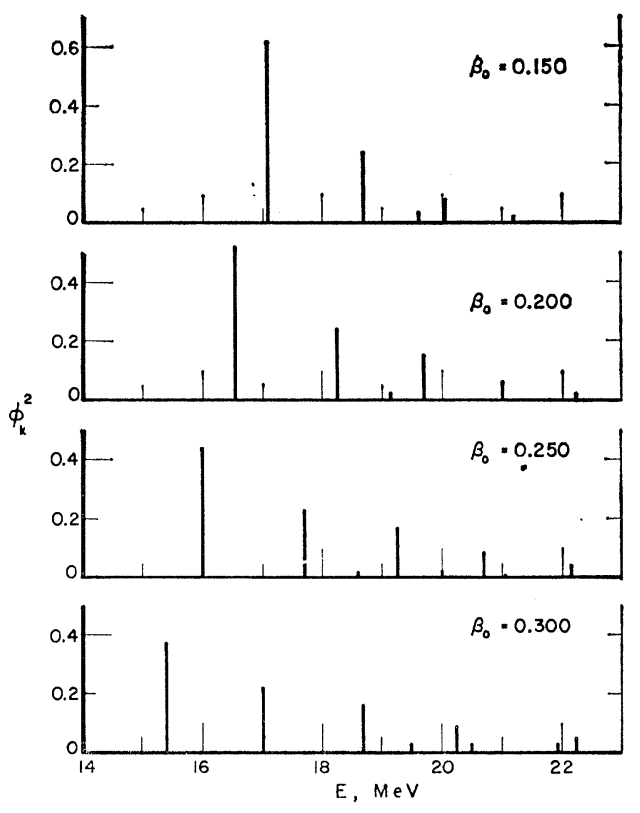

Fig. 21. Dipole strengths $\varphi_{\mathbf{k}}{ }^{2}$ for different values of $\beta_{0}$ $\left(E_{1}=18.0 \mathrm{MeV}, E_{2}=1.2 \mathrm{MeV}, N_{\text {phonon }}=8\right)$.

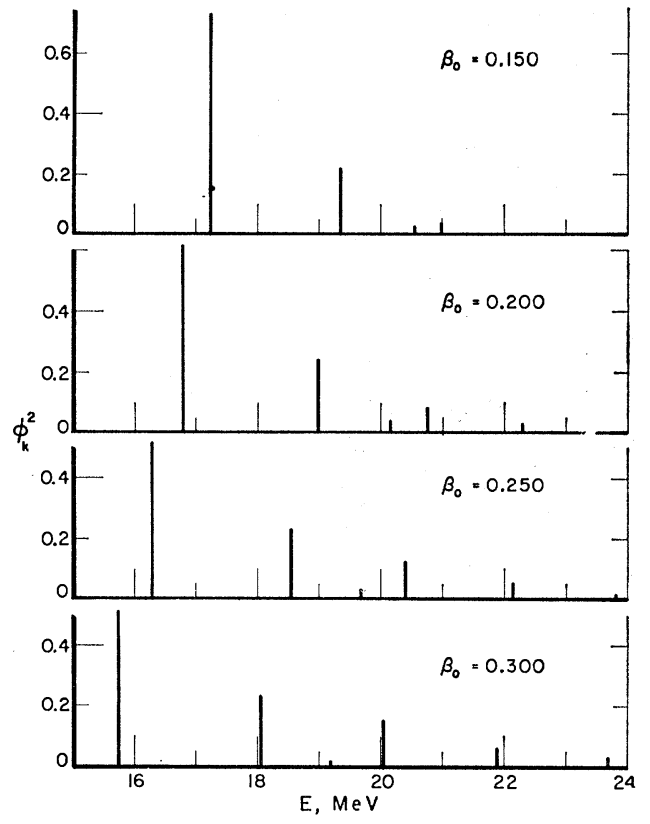

FIG. 22. Dipole strengths $\varphi_{\mathbf{k}}{ }^{2}$ for different values of $\beta_{0}$ $\left(E_{1}=18.0 \mathrm{MeV}, E_{2}=1.6 \mathrm{MeV}, N_{\text {phonon }}=8\right)$.

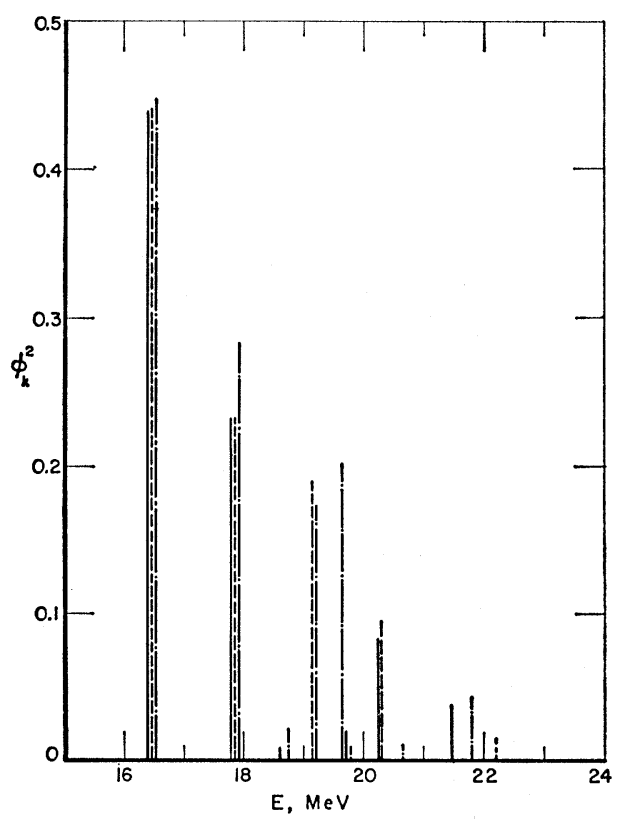

Fig. 23. Dipole strengths for different numbers of quadrupole phonons admixed to the giant dipole state $\left(E_{1}=18.0 \mathrm{MeV}, E_{2}=1.0\right.$ $\mathrm{MeV}, \beta_{0}=0.20$; straight line, $N_{\text {phonon }}=8$; dashed line, $N_{\text {phonon }}=6$; dot-dash line, $N_{\text {phonon }}=4$ ).

grouping into 1-ph states. However, it seems that in fact the phonon character is retained to some approximation; otherwise it would be very difficult to explain the qualitative agreement of the harmonic approximation with the experimental cross sections. In any case, to obtain a more quantitative agreement between experiment and theory, it will be necessary first to give 
a sufficiently accurate description of the low-energy collective spectrum -at least as far as the $0^{+}$and $2^{+}$ states are concerned, which alone can participate in the dipole states. Then one may hope to achieve quite a good description when adding the dipole excitation since, as already mentioned, the two modes contain to a large extent different single-particle states.

The most conspicious discrepancy between theory and experiment is, however, the structure at the lowenergy side of the resonance. No consistent explanation of this discrepancy has as yet been given. It seems very likely that the excess cross section should be associated with some of those states which in the schematic model ${ }^{26}$ have been swept clear of any transition strength. ${ }^{27}$

${ }^{26}$ G. E. Brown and M. Bolsterli, Phys. Rev. Letters 3, 472
In the language of the collective model the giant resonance is an isospin wave. It can be coupled to the spin wave. This would result in a splitting of the giant resonance, as observed in the calculations concerning $\mathrm{O}^{16}$ where two states carry appreciable dipole strength, the upper being the spin-flip state. It is possible that the same coupling would lead to a structure on the lowenergy side of the giant resonance. However, it is very unlikely that this structure would be as complicated as that in praseodymium, Fig. 11. This point thus merits a quantitative exploration.

(1959); G. E. Brown, Modified Theory of Nuclear Models (NorthHolland Publishing Company, Amsterdam, 1964).

${ }^{27}$ M. Danos and E. G. Fuller, Ann. Rev. Nucl. Sci. 15, 29 (1965)

\title{
Properties of the Projected Spectra for Finite Nuclei
}

\author{
Chindhu S. Warke \\ Tata Institute of Fundamental Research, Bombay, India \\ AND \\ M. R. GUNYE \\ Atomic Energy Establishment, Trombay, Bombay, India
}

(Received 16 September 1966)

\begin{abstract}
The general features of the expressions useful for numerical calculations of the projected deformed HartreeFock $(\mathrm{HF})$ spectra for finite nuclei are investigated. It is proved that the projected deformed $\mathrm{HF}$ wavefunction gives the possible nuclear spins as $I=0,2,4, \ldots, I_{\max }$ for a $K=0$ band and $I=K, K+1, \ldots, I_{\max }$ for a $K \neq 0$ band. It is further proved that if the energy $E_{I=K}$ of the projected $I=K$ state is greater (less) than the HF energy $E_{K} \mathrm{HF}$, then $E_{I}$ is greater (less) than $E_{I}{ }^{\prime}$ for $I<I^{\prime}$. A plausible reason why one should use the deformed HF state rather than any other deformed state is also pointed out.
\end{abstract}

\section{INTRODUCTION}

$\mathrm{R}^{\mathrm{H}}$ ECENTLY there has been considerable interest in Hartree-Fock (HF) calculations for finite nuclei. In nuclear HF calculations there is a special difficulty due to the nucleon-nucleon interaction inside the finite nucleus. Various different approaches are suggested in the literature to cope with this difficulty. ${ }^{1}$ Here we will not be concerned with this aspect of the problem. We simply assume some effective internucleon potential inside the nucleus. In the literature, two types of $\mathrm{HF}$ calculations are reported: (1) radial $\mathrm{HF}$ calculations for nearly closed-shell nuclei, ${ }^{2}$ and (2) deformed HF calculations for nonspherical nuclei.

1 K. A. Brueckner, J. L. Gammel, and H. Weitzner, Phys. Rev. 110, 431 (1958); S. A. Moszkowski and B. L. Scott, Ann. Phys. (N. Y.) 11, 657 (1960); R. K. Bhaduri and E. L. Tomnsiak, Proc. Phys. Soc. (London) 86, 451 (1965); C. Shakin and Y. R. Waghmare, Phys. Rev. Letters 16, 403 (1966).

2 Nazakat Ullah and R. K. Nesbet, Nucl. Phys. 39, 239 (1962); 46, 254 (1963); Phys. Rev. 134, B308 (1964); R. Muthukrishnan and M. Baranger, Phys. Letters 18, 160 (1965); A. K. Kerman, J. P. Svenne, and F. M. H. Villars, Phys. Rev. 147, 710 (1966).
Following the finding that one can obtain nearly the same physical results for a nucleus by doing the intermediate coupling calculations or by doing the deformed $\mathrm{HF}$ calculations and then projecting good angular momentum states from it, deformed HF calculations gained popularity. ${ }^{3}$ By deformed HF calculations, we mean those in which the radial parts of the single-particle orbitals are taken as harmonic-oscillator radial wave functions while the angular momentum parts are determined from the $\mathrm{HF}$ variational calculation. Here we will be dealing with deformed $\mathrm{HF}$ calculations only.

We investigate the general broad features of the low-lying excited states of nuclei as obtained by projecting the good angular momentum states from the deformed HF wave function, and we derive the properties of the projected spectrum. We also give a justification of why one should project from the HF state

${ }^{3}$ M. Redlich, Phys. Rev. 110, 468 (1958); D. Kurath and L. Picman, Nucl. Phys. 10, 313 (1959); W. H. Bassichis, B. Giraud, and G. Ripka, Phys. Rev. Letters 15, 980 (1965). 